\title{
Probing Nanoparticle Effect in Protein-Surfactant Complexes
}

\author{
Sumit Mehan ${ }^{1}$, V.K. Aswal ${ }^{1, *}$ and J. Kohlbrecher ${ }^{2}$ \\ ${ }^{1}$ Solid State Physics Division, Bhabha Atomic Research Centre, Mumbai 400 085, India \\ ${ }^{2}$ Laboratory for Neutron Scattering, Paul Scherrer Institut, CH-5232 PSI Villigen, Switzerland \\ "E-mail:vkaswal@barc.gov.in
}

\begin{abstract}
SANS experiments have been carried to probe the role of anionic silica nanoparticles in the anionic BSA protein-cationic DTAB surfactant complexes. In protein-surfactant complex, surfactant molecules aggregate to form micelle-like clusters along the unfolded polypeptide chains of the protein. The nanoparticle aggregation mediated by oppositely charged protein-surfactant complex coexists with the free protein-surfactant complexes in the nanoparticle-protein-surfactant system. There is rearrangement of micelles in adsorbed protein-surfactant complex on nanoparticles in leading to their (nanoparticle) aggregation. On the other hand, the unfolding of protein in free protein-surfactant complex is found to be significantly enhanced in presence of nanoparticles.
\end{abstract}

Keywords: Nanoparticles, nanoparticle-protein interaction, protein-surfactant complexes, small-angle neutron scattering PACS: 78.67.Bf, 87.15B-, 61.05.fg

\section{INTRODUCTION}

Nanoparticles due to their small size and large surface-to-volume ratio possess unique and distinct properties which are useful for their numerous applications [1]. Many of these applications require interaction of nanoparticles with macromolecules such as proteins [2]. In particular, in the field of nanobiotechnology, nanoparticle-protein complexes has gained sufficient interest because they can access to and operate within the cell due to their size comparable to that of cell. The nanoparticle-proteinsurfactant complexes are used in food, medicine etc., where three components in varying proportions coexist with each other. The protein-surfactant complex provides the advantage of effective tuning of adsorption behavior with nanoparticles by varying concentration ratio of protein to surfactant or charge of surfactant molecules. Therefore, it is interesting to study the interactions in these complexes and tune them for various potential applications [3].

Herein, we have examined the effect of addition of anionic silica nanoparticle in the anionic BSA protein-cationic DTAB surfactant complex. It is expected that the presence of nanoparticle to enhance the protein-surfactant interaction as well as proteinsurfactant complex to modify the nanoparticle interaction. The structure and interaction in these systems have been studied by small-angle neutron scattering (SANS). The technique of SANS is an ideal probe to study both structure and interaction of such multi-component systems under native environment [4]. This technique is used for various kinds of nanostructure materials in a length scale of $1-100 \mathrm{~nm}$. The fact that the neutron scattering is different for solvent $\mathrm{H}_{2} \mathrm{O}$ and $\mathrm{D}_{2} \mathrm{O}$, these have been used selectively to contrast match components wherever required to simplify the scattering from complex systems.

\section{EXPERIMENT}

Electrostatically stabilized ludox HS40 silica nanoparticles, BSA protein and dodecyl trimethyl ammonium bromide (DTAB) surfactant were purchased from Sigma-Aldrich. The stock solutions of each component were prepared by dissolving the required weighted amounts of components in $20 \mathrm{mM}$ phosphate buffer $(\mathrm{pH}=7)$ in presence of $0.2 \mathrm{M} \mathrm{NaCl}$. The salt is used to reduce the electrostatic interaction between different components. The samples were prepared in $\mathrm{D}_{2} \mathrm{O}$ which provides better contrast in neutron scattering experiments for hydrogenous components (e.g. BSA, DTAB). The mixed solvent of $\mathrm{D}_{2} \mathrm{O}$ and $\mathrm{H}_{2} \mathrm{O}(60 \%$ D2O) was used for contrast matching nanoparticles. The silica nanoparticle interaction with protein and surfactant is studied at fixed concentrations of nanoparticle (1 wt $\%)$ and protein $(1 \mathrm{wt} \%)$ with varying surfactant concentration $(10$ - 50 mM). Small-angle neutron scattering experiments were performed at the SANS-I facility, Swiss Spallation Neutron Source SINQ, Paul Scherrer Institute, Switzerland [5]. Data were collected over a 
wave vector transfer $(Q=4 \pi \sin (\theta / 2) / \lambda$, where $\theta$ is scattering angle) range of 0.005 to $0.30 \AA^{-1}$. Data were corrected for background and empty cell, and normalized to absolute unit of cross-section using standard procedures.

\section{SANS ANALYSIS}

In SANS experiments, one measures differential scattering cross-section per unit volume $d \Sigma / d \Omega$ as a function of $Q$, which can be expressed as [4]

$$
\frac{d \Sigma}{d \Omega}(Q)=n V_{p}^{2} \Delta \rho^{2} P(Q) S(Q)+B
$$

where $n$ is number density of particles, $V_{p}$ is particle volume and $\Delta \rho^{2}$ is scattering contrast of particles. $P(Q)$ and $S(Q)$ are the intraparticle and interparticle structure factors, respectively. $P(Q)$ depends on the shape and size of particles, whereas $S(Q)$ is decided by the interparticle interaction. $B$ is the incoherent background, which predominately arises due to presence of hydrogen in the sample.

For a sphere of radius $R, P(Q)$ is given by

$$
P(Q)=\left[\frac{3\{\sin (Q R)-(Q R) \cos (Q R)\}}{(Q R)^{3}}\right]^{2}
$$

$S(Q)$ of mass fractal structure for protein-surfactant complex in modeling the bead-necklace structure of adsorbed micelles along the unfolded protein chain is given by

$$
S_{f}(Q)=1+\frac{1}{\left(Q r_{0}\right)^{D}} \frac{D \Gamma(D-1)}{\left[1+\frac{1}{(Q \xi)^{2}}\right]^{D-1 / 2}} \sin \left\{(D-1) \times \tan ^{-1}(Q \xi)\right\}
$$

where $D$ is fractal dimension, $\xi$ is the overall size of a fractal aggregate and $r_{0}$ is characteristic dimension of individual particles. $\Gamma$ represents a mathematical gamma function.

The strong interaction between oppositely charged protein-surfactant complex with nanoparticles is known to result in aggregation of nanoparticles. The aggregates are characterized by a mass fractal structure.

\section{RESULTS AND DISCUSSION}

SANS data of $1 \mathrm{wt} \%$ BSA in presence of varying DTAB concentration are shown in Figure 1. The features of the scattering data are very different from that of the sum of the individual of components. One of the interesting features is the linearity of the scattering profiles on log-log scale in the intermediate $Q$ range with a $Q$ range of linearity increasing with surfactant concentration. This is an indication of formation of fractal structure by the protein-surfactant complex [6]. The fractal structure of the complex on the basis of the necklace model considers micelle-like clusters of the surfactant formed along the unfolded polypeptide chain of the protein. The slope of the scattering data on log-log scale gives the value of the fractal dimension $D$ of the complex. The cut-offs of the linear range of the data at low and high $Q$ values are, respectively, related to the formation of the extent of the complex and the size of the individual micelles in the complex. The fitted parameters of the analysis are given in Table 1. It is found that the fractal dimension $(D)$ decreases on increasing surfactant concentration. The size of micelle-like clusters $(R)$ does not change whereas overall size of the complex $(\xi)$ increases with increase in surfactant concentration.

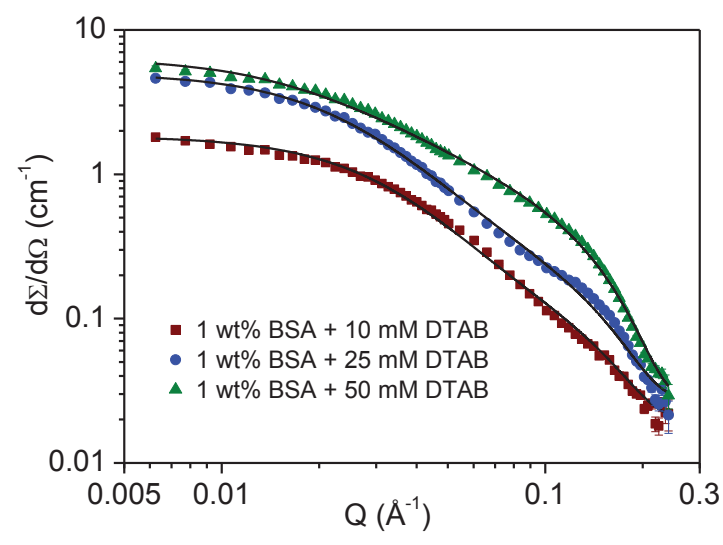

FIGURE 1. SANS data of $1 \mathrm{wt} \%$ BSA protein with varying concentration of DTAB surfactant.

TABLE 1. Fitted parameters of complexes of $1 \mathrm{wt} \%$ BSA protein with varying concentration of DTAB surfactant.

\begin{tabular}{cccc}
\hline$[\mathrm{DTAB}]$ & $D$ & $R(\AA)$ & $\xi(\AA)$ \\
\hline $10 \mathrm{mM}$ & 2.7 & 16.8 & 25.5 \\
$25 \mathrm{mM}$ & 2.4 & 16.8 & 37.7 \\
$50 \mathrm{mM}$ & 1.6 & 16.8 & 61.8 \\
\hline
\end{tabular}

Figure 2 shows SANS data of effect of addition of nanoparticle in protein-surfactant system. The data show a very strong build-up of scattering in the low Q region followed by the features that of the proteinsurfactant complex in the intermediate to high $Q$ region. The nanoparticles are found to be aggregated in this system as appeared from the observed turbidity of the system. Therefore, the build-up of scattering in the low Q region with linear behaviour on log-log scale suggests fractal aggregates of nanoparticles. The aggregates could be formed through the oppositely charged surfactant micelles or protein-surfactant complex mediation of nanoparticles [7]. It is observed that the data in the low $Q$ region corresponding to the nanoparticle aggregation remain more or less same with the increase in surfactant concentration. There are systematic changes in the higher $Q$ region, which are similar to the protein-surfactant system. This suggests 
irrespective of absence or presence of nanoparticles, the interaction of protein and surfactant is maintained over a wide concentration range of surfactant.

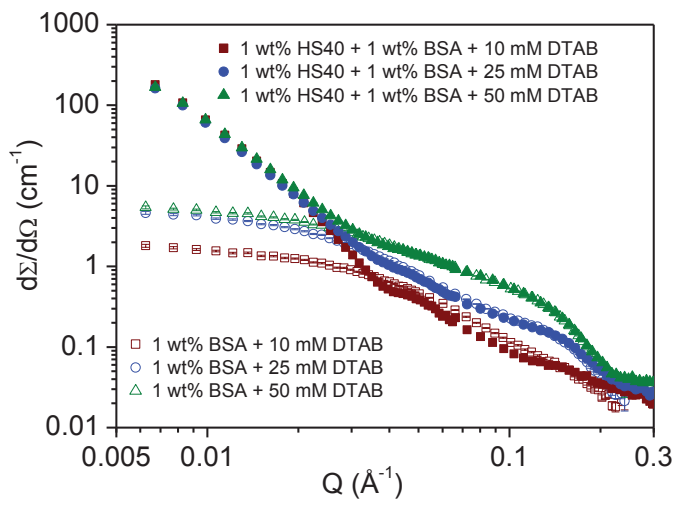

FIGURE 2. SANS data of $1 \mathrm{wt} \%$ BSA protein with varying concentration of DTAB surfactant in presence of $1 \mathrm{wt} \%$ HS40 nanoparticles. For comparison data without nanoparticles are also shown.

Figure 3 shows SANS data of nanoparticle in protein-surfactant system for the solution condition where nanoparticles are contrast matched. The scattering profile of nanoparticle-protein-surfactant system clearly shows very different profile than protein-surfactant system. The build-up of scattering in low $Q$ region is due to the shell formation of micelle-like clusters of protein-surfactant complex around non-visible nanoparticles in these aggregates. The scattering profile of nanoparticle in protein-surfactant system is also compared with nanoparticle-surfactant system (nanoparticles contrast matched) in the inset of figure 3. The scattering profile of nanoparticle in protein-surfactant system is considerably different than that of nanoparticlesurfactant system. The data are different in low $Q$ region because of differences in the fractal dimension of two systems, whereas the differences in intermediate $Q$ range arise because of different structures (micelles versus protein-surfactant complex) coexisting with nanoparticle aggregates. The data are fitted with a shell of protein-surfactant complex around the non-visible nanoparticles in their aggregates coexisting with free protein-surfactant complexes. The shell thickness is found to be around $43 \AA$. The similar values of shell thicknesses for nanoparticle in protein-surfactant and nanoparticlesurfactant systems indicate the rearrangement of micelles in adsorbed protein-surfactant complex on nanoparticles in leading to their (nanoparticle) aggregation in nanoparticle with protein-surfactant system. Moreover, the unfolding of protein in free protein-surfactant complex in the three-component system is found to be enhanced by the presence of nanoparticles (Table 2). This in turn suggests that the structure in nanoparticle with protein-surfactant complexes is determined by the modified interactions of individual components.

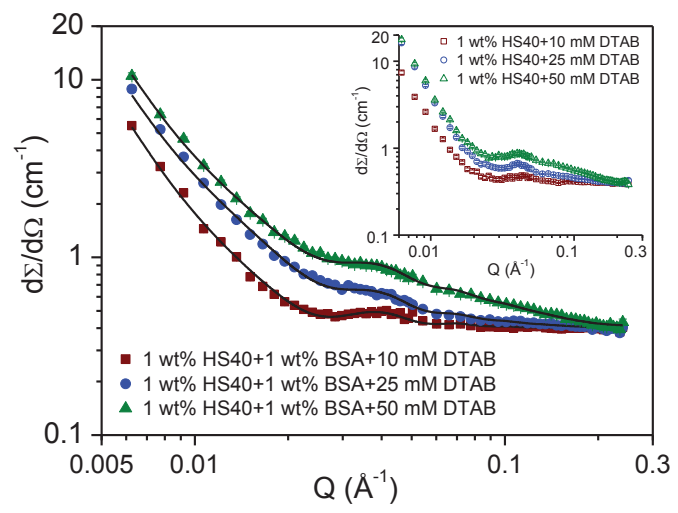

FIGURE 3. SANS data of $1 \mathrm{wt} \%$ BSA protein with varying concentration of DTAB surfactant in presence of $1 \mathrm{wt} \%$ HS40 contrast-matched nanoparticles. Inset shows the data of nanoparticle-surfactant system without protein.

TABLE 2. Fitted parameters of nanoparticle effect ( $1 \mathrm{wt} \%$ HS40) on complexes of $1 \mathrm{wt} \%$ BSA protein with varying concentration of DTAB surfactant.

\begin{tabular}{cccc}
\hline$[$ DTAB $]$ & $D$ & $R(\AA)$ & $\xi(\AA)$ \\
\hline $10 \mathrm{mM}$ & 2.2 & 16.8 & 34.3 \\
$25 \mathrm{mM}$ & 2.0 & 16.8 & 59.5 \\
$50 \mathrm{mM}$ & 1.3 & 16.8 & 70.0 \\
\hline
\end{tabular}

In summary, the nanoparticle effect in proteinsurfactant complex show an interesting phase behaviour where nanoparticle aggregates coexist with the protein-surfactant complexes. Both the nanoparticle aggregates and protein-surfactant complexes are characterized by the mass fractal at different length scales. The protein-surfactant interaction is enhanced in presence of nanoparticles.

\section{REFERENCES}

1. I. Lynch and K.A. Dawson, Nano Today 3, 40 (2008).

2. S. Kumar, V.K. Aswal and J. Kohlbrecher, Langmuir 27, 10167-10173 (2011).

3. S. Mehan, A. Chinchalikar, S. Kumar, V.K. Aswal and R. Schweins, Langmuir 29, 11290-11299 (2013).

4. D. Svergun and M. H. J. Koch, Rep. Prog. Phys. 66, 1735-1782 (2003).

5. J. Kohlbrecher and W. Wagner, J. Appl. Crystallogr. 33, 804-806 (2000).

6. V.K. Aswal, S. Chodankar, V. Ronny, J. Kohlbrecher and A.G. Wagh, Phys. Rev. E 80, 011924 (2009).

7. S. Kumar, V.K. Aswal and J. Kohlbrecher, Langmuir 28, 9288-9297 (2012). 